\title{
Características psicológicas de pacientes submetidos a cirurgia bariátrica
}

\author{
Psychological characteristics of patients submitted to bariatric surgery
}

\begin{abstract}
Ronis Magdaleno Jr. ${ }^{1}$, Elinton Adami Chaim², Egberto Ribeiro Turato ${ }^{3}$
${ }^{1}$ Psiquiatra, psicanalista. Membro associado da Sociedade Brasileira de Psicanálise de São Paulo. Doutorando em Saúde Mental, Departamento de Psicologia Médica e Psiquiatria, Faculdade de Ciências Médicas, Universidade Estadual de Campinas (UNICAMP), Campinas, SP. ${ }^{2}$ Doutor. Médico cirurgião, responsável pelo Serviço de Cirurgia Bariátrica, Hospital de Clínicas, UNICAMP. ${ }^{3}$ Professor livre-docente. Médico psiquiatra. Coordenador do Laboratório de Pesquisa Clínico-Qualitativa, Faculdades de Ciências Médicas, UNICAMP.

Este estudo foi realizado no Ambulatório de Cirurgia Bariátrica do Hospital de Clínicas da Universidade Estadual de Campinas (UNICAMP), e sua execução contou com a colaboração do Laboratório de Pesquisa Clínico-Qualitativa da Faculdade de Ciências Médicas da UNICAMP, coordenado pelo Prof. Dr. Egberto Ribeiro Turato. É parte de projeto de pesquisa de doutorado na área se Saúde Mental, devidamente aprovado pelo Comitê de Ética em Pesquisa da Faculdade de Ciências Médicas, UNICAMP (parecer $n^{\circ}$ 534/2006). Título do projeto: "Vivências emocionais de mulheres submetidas à cirurgia bariátrica no HC-UNICAMP: um estudo clínico-qualitativo".
\end{abstract}

\section{Resumo}

Introdução: $\mathrm{O}$ grande número de pacientes submetidos a cirurgia bariátrica e que, no pós-operatório, apresentam complicações psicológicas e psiquiátricas justifica uma investigação pré-operatória acurada, bem como categorização daqueles que se submeterão ao procedimento cirúrgico, visando predizer eventuais complicações e individualizar condutas psicológicas que possam favorecer a adesão do paciente. A avaliação psicodinâmica pode fornecer elementos para tal categorização e, assim, propor estratégias de abordagem pré e pós-operatória eficazes. Procuramos identificar estruturas de personalidade que possam orientar o acompanhamento pós-operatório, bem como critérios auxiliares de inclusão/exclusão do procedimento cirúrgico.

Método: Relato de pesquisa empírica conduzida em atendimentos a pacientes submetidos a cirurgia bariátrica, em grupo terapêutico aberto.

Discussão: Pacientes, depois de operados, podem passar por determinadas fases de reestruturação emocional, como uma primeira fase de triunfo, seguida de fase de risco para surgimento de quadros melancólicos e de novas adições. Identificamos três categorias estruturais psicológicas: estrutura melancólica, cujos pacientes parecem ter maior possibilidade de desenvolver outras condutas aditivas no pós-operatório, sobretudo alimentares, por não suportarem a frustração pela perda; estrutura desmentalizada, na qual, por faltar uma capacidade elaborativa, o paciente não consegue reorganizarse frente ao desafio de permanecer com peso controlado; e, finalmente, a estrutura perversa, cujos sujeitos mantêm a programada perda de peso, porém a custas de comportamentos que levam desconfortos à equipe de saúde. Estabelecer categorias psicológicas classificatórias pode ser crucial para que se proponham condutas no pós-operatório, inclusive indicação de psicoterapia com especialista, visando individualizar o atendimento, incrementando sucesso terapêutico específico.

Descritores: Obesidade mórbida, cirurgia bariátrica, psicanálise, psicologia médica, psicoterapia de grupo.

\begin{abstract}
Introduction: The great number of patients submitted to bariatric surgery who have psychological and psychiatric complications during the postoperative period require a thorough preoperative investigation and a categorization with the purpose of predicting possible complications and personalizing psychological care that might favor patient compliance. Psychodynamic assessment may provide data for such categorization and, thus, suggest effective pre- and postoperative approach strategies. Therefore, the objective of this study was to identify personality structures that may be useful in the postoperative follow-up, as well as additional inclusion and exclusion criteria for the surgical procedure.

Methods: Report of empirical survey conducted during psychotherapy sessions with an open group of patients who underwent bariatric surgery.

Discussion: After surgery, patients may experience phases of emotional restructuring, such as an initial phase of feeling triumphant, followed by a phase when there is risk of melancholic behavior and new addictions. We identified three categories of psychological structures: melancholic structure (patients seem to be more likely to develop other postoperative addictive behaviors, mainly eating disorders, since they cannot tolerate the frustration of the loss); dementalized structure (due to the lack of elaborative capacity, patients are unable to reorganize themselves in face of the challenge of keeping their weight under control); and perverse structure (patients comply with the scheduled weight loss; however, their behavior makes the health team experience uncomfortable situations). Establishing psychological categories may be crucial in order to suggest postoperative management strategies, including referral to a psychotherapist with the purpose of providing personalized care, thus increasing specific therapeutic success.
\end{abstract}

Keywords: Morbid obesity, bariatric surgery, psychoanalysis, medical psychology, group psychotherapy.

\section{Correspondência:}

Ronis Magdaleno Jr., Rua Padre Almeida, 515/14, CEP 13025-251, Campinas, SP. Tel.: (19) 3254.2103, Fax: (19) 3203.2103. E-mail: ronism@uol.com.br

Não há conflitos de interesse associados à publicação deste artigo.

Copyright (C) Revista de Psiquiatria do Rio Grande do Sul - APRS

Recebido em 22/04/2008. Aceito em 05/11/2008. 


\section{Introdução}

A obesidade é uma doença crônica, de prevalência crescente, que, pelos riscos associados, vem sendo considerada como um dos principais problemas de saúde pública da sociedade moderna ${ }^{1}$. Além dos problemas que afetam diretamente a qualidade de vida do portador, a obesidade acarreta um aumento na incidência de vários outros quadros patológicos.

Segundo relatório de 2006 da Organização Mundial de Saúde ", "a obesidade alcançou globalmente proporções epidêmicas, com mais de um bilhão de adultos com sobrepeso - pelo menos 300 milhões deles clinicamente obesos - e é a maior responsável pelo aumento global de desabilidades e doenças crônicas". Esses dados têm alertado as autoridades e requerido grandes esforços por parte dos médicos e outros profissionais de saúde no sentido de encontrar meios de controle e tratamento para a obesidade.

O grande problema dos tratamentos propostos para a obesidade mórbida é a manutenção da perda do peso a longo prazo, e a cirurgia bariátrica surgiu como ferramenta terapêutica eficaz, com reais possibilidades de minimizar as falhas terapêuticas que ocorriam com os tratamentos clínicos e nutricionais ${ }^{2,3}$.

A cirurgia bariátrica foi recomendada durante a Conferência de Desenvolvimento de Consenso do National Institutes of Health (NIH) de $1991^{3}$, para indivíduos bem informados, motivados e com obesidade de classe 3, que tivessem riscos operatórios aceitáveis, e para aqueles com obesidade de classe 2 e condições pré-mórbidas de alto risco. Aconselhou-se ainda, como fundamental, uma seleção cuidadosa de candidatos à cirurgia por equipe multidisciplinar.

Segal \& Fandiño ${ }^{4}$, em concordância com a recomendação do NIH, avaliaram que "deve ficar clara a necessidade de avaliação clínica, laboratorial e psiquiátrica de forma regular nos períodos pré e pós-operatório", mas advertem que tem se observado "um crescente abandono de critérios psicológicos na seleção de candidatos a esses procedimentos, provavelmente devido a ausência de instrumentos que permitam adequada acurácia prognóstica, mostrando um julgamento clínico baseado em evidências, cada vez menos objetivo".

Um levantamento feito por Appolinário ${ }^{5}$ mostra que estudos de seguimento pós-operatório de longo prazo de pacientes submetidos a cirurgia bariátrica "reportaram várias condições psiquiátricas como causas de morte no pós-operatório, sendo o suicídio a principal ocorrência". Omalu et al. ${ }^{6}$ apresentam relatos de casos de suicídio de pacientes previamente depressivos após a realização da cirurgia bariátrica. Esses achados apontam para um desequilíbrio no jogo de forças psíquicas no pós-operatório de tais sujeitos, e que os novos sintomas que surgem são basicamente de caráter autoagressivo. Leal \& Baldin ${ }^{7}$ apresentam relatos de casos onde um incremento da agressividade é referido pelas pacientes após a cirurgia.

A preocupação desses autores é pertinente, posto que o procedimento cirúrgico tem sido indicado e realizado com frequência cada vez maior, e os cuidados com as consequências psicológicas e psiquiátricas, ainda pouco conhecidas, dessa prática não estão bem definidos.

Kalarchian et al. ${ }^{8}$, após consistente investigação de transtornos psiquiátricos, tanto do eixo I como do eixo II do Manual de Diagnóstico e Estatística de Distúrbios Mentais, $4^{\mathrm{a}}$ edição (DSM-IV) ${ }^{9}$, concluíram que há "evidências contundentes de que transtornos psiquiátricos são uma preocupação maior para a referida população de pacientes, não apenas por serem relativamente comuns, mas porque também estão associados à gravidade da obesidade e à condição de saúde funcional diminuída".

Magdaleno et al. ${ }^{10}$ demonstraram que, apesar das agudas modificações físicas e psíquicas que a cirurgia bariátrica impõe, a aceitação social e o sentimento de reencontro com sua identidade que estava encoberta pelo excesso de gordura corporal, rompe o ciclo vicioso baixa autoestima-incremento da ansiedade-impulso alimentar, com sensível melhora da qualidade de vida dos pacientes ${ }^{10}$. Do ponto psicodinâmico, a obesidade corresponde a uma estrutura como efeito final de uma complexa trama de dificuldades psíquicas e sua interação com o meio, que parte de problemas em fases precoces do desenvolvimento do ser humano ${ }^{11,12}$.

Sabe-se, desde Freud ${ }^{13,14}$, que, nas fases iniciais do desenvolvimento, o ódio da criança em relação ao ambiente que a frustra é predominante, e os ataques que a criança faz são intensos, podendo, caso a resposta do ambiente seja desfavorável, danificar o sistema relacional dessa criança com os outros, transtornando suas funções psíquicas básicas. A função simbólica da incorporação expressa pela alimentação é, nesse período, o modelo do qual a criança dispõe para relacionar-se com o mundo. A partir de falhas nesse momento fundamental da construção do sistema relacional da criança se estruturariam os sintomas mais primitivos que irão se expressar, mais tarde, por comportamentos aditivos, entre eles a obesidade mórbida. A incapacidade para criar um referencial simbólico que seja suficiente para dar algum destino para a forte pressão de impulsos primitivos deixa em aberto a via da descarga corporal imediata destes.

Segundo Mahler ${ }^{11}$, a identidade coesa da imagem corporal da criança fundamenta-se em suas experiências iniciais nos campos sensorial e motor, experimentados na relação fusional com a mãe. A criança aprende e apreende o mundo por intermédio dessa relação. É uma fase na qual o Eu se forma a partir da imagem da mãe. Winnicott ${ }^{15}$ chamou esse momento do desenvolvimento de "fase do espelho", no qual a criança deve poder reconhecer a si mesma no olhar da mãe, e a partir daí iniciar o processo de formação de sua identidade. Falhas nesses momentos iniciais da formação do Eu obrigam o bebê a se defender, estruturando áreas deformadas no psiquismo que o incapacitariam para lidar com a pressão vinda do corpo.

Ainda segundo Winnicott ${ }^{16}$, no decorrer do desenvolvimento normal de um bebê, a "mãe devota suficientemente boa" acolhe o bebê em seu "ambiente interno" e o protege 
das tumultuadas tensões internas e externas, proporcionando, assim, uma progressiva diferenciação do soma da criança em direção a formação de um psicossoma, que fará a intermediação entre as intensas exigências vindas de dentro e as exigências do mundo externo. Assim, o psicossoma seria a estrutura que se forma a partir da relação do corpo com suas pulsões e com o ambiente. Caso haja alguma deficiência nesse processo de maturação, partes desse corpo continuam funcionando de forma primitiva, sem intermediação organizadora, ficando submetidas à descarga imediata da tensão. Tustin ${ }^{12}$, a partir do estudo de crianças com autismo psicogênico, postula a existência de áreas isoladas da personalidade de adultos com funcionamento autista, que seriam a base estrutural de transtornos mentais como as adições, transtornos alimentares, e obesidade mórbida, entre eles.

O problema relevante que se apresenta é que a retirada ou o impedimento cirúrgico do sintoma cria um campo novo de estudo da dinâmica biopsicológica do sujeito, pois a angústia que, de algum modo, se resolvia através do sintoma tem essa via impedida - aguda e artificialmente - de modo quase que absoluto após a cirurgia bariátrica. Nosso objetivo é, a partir da compreensão das experiência vividas pelos pacientes operados, definir algumas estratégias para o manejo com os pacientes e com a equipe de profissionais de saúde envolvida com a realização da cirurgia bariátrica.

\section{Método}

O presente trabalho é resultado de reflexões clínicopsicológicas provenientes do processo de aculturação do pesquisador em campo e refere-se a um projeto de doutorado, em andamento, na área de saúde mental, com sujeitos que foram submetidos a cirurgia bariátrica no Serviço de Cirurgia Bariátrica do Hospital das Clínicas da Universidade Estadual de Campinas (UNICAMP), Campinas (SP). A aculturação é procedimento usual em investigações qualitativas com referencial humanístico e visa à construção de amostra representativa em pesquisa no enfoque metodológico clínico-qualitativo ${ }^{17}$.

Este artigo traz um relato de observação empírica no atendimento psicológico a pacientes submetidos a cirurgia bariátrica no Hospital de Clínicas da UNICAMP, a partir da aplicação do método clínico em grupo terapêutico aberto. Entendemos por método clínico aquele que procura se adequar aos problemas originados na observação clínica, a partir de instrumentos relacionados à prática clínica, apoiado no empirismo e na teoria ligada à atuação clínica ${ }^{18}$. Assim, a origem do método é a atitude clínica do pesquisador, baseada "na acolhida do sofrimento existencial e emocional do indivíduo alvo dos estudos do pesquisador"17.

Arruda $^{18}$ complementa que a atitude clínica, somada ao emprego do modelo interpretativo das ciências humanas, possibilita a "compreensão, a conexão de sentidos e a elaboração de conhecimentos". Tais autores enfatizam, portanto, a possibilidade de um tratamento qualitativo ao processo de pesquisa e à análise dos dados obtidos quando utilizamos o método clínico ${ }^{19}$, e que, portanto, esse procedimento situa-se entre as ferramentas científicas de investigação, sendo válidos os resultados obtidos.

Utilizamo-nos da observação clínica dos pacientes em grupos terapêuticos abertos. Esses grupos são semanais, conduzidos por psicólogos do Ambulatório de Cirurgia Bariátrica do Hospital de Clínicas da UNICAMP e baseiamse no convite e na participação espontânea dos pacientes que foram submetidos à cirurgia. $\mathrm{O}$ enfoque terapêutico do grupo é amplo, visando esclarecimentos a respeito de problemas práticos que surgem no pós-operatório, identificação de conflitos, compreensão da dinâmica psíquica dos pacientes e, por fim, a possibilidade de um melhor manejo, pelos pacientes, do desafio que representa o pósoperatório da cirurgia. Tem como objetivo principal situar os pacientes na sua nova condição de vida e, com isso, melhorar a adesão ao tratamento e instrumentalizá-los para manter a perda de peso.

A partir do material que surgiu espontaneamente nos grupos, daquilo que pudemos identificar da dinâmica grupal e na discussão com os pares no Laboratório de Pesquisa Clínico-Qualitativa, pudemos identificar alguns fenômenos que são constitutivos da realidade psíquica de tais sujeitos e que acreditamos ser fenômenos comuns no pós-operatório da cirurgia bariátrica.

\section{Discussão}

\section{Sobre a experiência clínica no grupo de pós-operatório}

...mas para nós isto tudo é muito difícil, pois a gente pensa com o corpo.

O sujeito que chega ao grupo de pós-operatório já se apresenta de algum modo como um vencedor. Venceu os desafios do período pré-operatório, sobreviveu à cirurgia, suportou o primeiro mês de operado, durante o qual a dieta líquida e pastosa é sempre vivida como "um suplício a ser tolerado por uma causa maior" (frase de uma das pacientes do grupo). Contudo, tal sensação de vitória, pouco a pouco, vai sendo encoberta pela dureza da condição à qual esses sujeitos se expõem. A alimentação, que era anteriormente vivida como prazer incondicional, salvo por certa culpa pelas quantidades de alimento ingeridas, passa a ser um problema a ser enfrentado: o alimento "não desce bem", "enrosca", "fica parado", muitas vezes provoca vômito, dumping. O prazer em comer diminui muito. E agora, o que fazer com os indivíduos que tinham quase como prazer exclusivo em suas vidas o ato de comer? O que fazer com os sujeitos que "pensam com o corpo"?

É a partir deste momento que começam a se apresentar, verdadeiramente, para esses sujeitos os problemas que terão 
que enfrentar. Até o momento da cirurgia, por mais que tenham sido orientados e esclarecidos quanto às dificuldades, estavam, digamos, fascinados com a certeza de resolverem todos os seus problemas a partir da cirurgia ${ }^{20,21}$, como que num passe de mágica.

Uma situação clínica durante uma reunião do grupo pode ilustrar isso. Numa das reuniões discutíamos, como é frequente nesses grupos, o problema enfrentado pelo obeso em relação a sua dificuldade em se adequar aos padrões sociais. No meio da conversa, surge o comentário de um dos pacientes: "nosso problema são as catracas dos ônibus coletivos, se não prestar atenção, fica-se entalado". Tal fala cria um movimento imediato de concordância no grupo, que num clima de brincadeira começa a contar anedotas sobre a vida de cada um em relação às próprias experiências com catracas em ônibus. Certa euforia se instala, misturada com um alívio, porque praticamente todos agora podiam passar pelas referidas catracas dos ônibus.

O terapeuta intervém e diz "mas agora que todos já passam nas catracas, o que fazer?". Certo desconcerto se instala no grupo, uma angústia volta a se fazer presente. A fala do terapeuta remete o grupo à angústia subjacente ao triunfo que o paciente se esforça por sustentar: a ideia de que a cirurgia poderia, por si só, resolver todos os problemas da vida de cada um. A realidade, contudo, se mostra muito diversa disso. A vida não se resolve ao passar, metaforicamente, a catraca, ou concretamente, pela realização da cirurgia. A fala do terapeuta tem como objetivo estimular o pensamento, proporcionar a esses indivíduos que "pensam com o corpo" uma possibilidade de "pensar com a cabeça", refletir, expandindo suas capacidades elaborativas.

Ao passar por esse marco, que é a cirurgia, inicia-se uma fase de intensos desafios, talvez até maiores que antes, pois todo o sistema defensivo que era mantido em torno da alimentação e da obesidade fica impedido. O sujeito deve, rapidamente, aprender a lidar com suas angústias através de outros meios. Isso nem sempre é possível, deixando então abertos inúmeros canais patológicos. Pode-se dizer que a obesidade, vivida por todos como um grande problema, é também, no fundo, uma resposta para todos os problemas. Daí a conclusão óbvia que aparece tantas vezes: resolvendo o problema da obesidade, estará tudo resolvido. O momento em que tal fantasia se desvanece é crítico, e observamos que o sujeito fica susceptível às complicações emocionais e psiquiátricas que descreveremos a seguir.

\section{As vivências psicológicas no pós-operatório}

Uma primeira reação que se apresenta na quase totalidade dos pacientes é, como procuramos ilustrar com o material clínico acima, certo "triunfar" sobre a condição anterior de obesidade. É como se todo o problema houvesse sido derrotado pela cirurgia. Essa reação é intensamente reforçada pela perda de peso visível, pelo reforço positivo dado pela equipe, pelos familiares e pelos demais pacientes. Esse é um primeiro tempo definido pela própria equipe e pelos pacientes como de lua-de-mel, dentro do qual a perda de peso é tão evidente e o reforço positivo da equipe e dos pacientes é tão presente, que acaba compensando qualquer sofrimento. O paciente sente-se na "vitrine da moda", e todo aquele sofrimento advindo da exclusão social, da rejeição por ser gordo, da ferida narcísica que representa um corpo fora dos padrões, de repente se reverte.

Contudo, como toda lua-de-mel, tal período vai pouco a pouco terminando. No lugar dele, vão aparecendo outros sentimentos, que por vezes "parecem vir do nada": uma angústia, uma sensação de tédio indefinida, um vazio, algo que fica faltando, sentimentos de tristeza, uma vontade de ficar beliscando, o tempo todo, alguma coisa... mas ainda assim a perda de peso continua visível, o trauma físico da cirurgia vai cicatrizando, uma coisa equilibra a outra e a disposição de continuar lutando permanece.

A ameaça aparece quando a realidade de que o peso pode voltar a aumentar, apesar da cirurgia, se impõe. É nesse momento - e aqui já se passou 1 ano ou um pouco mais após a cirurgia - que o sintoma "obesidade", que foi impedido pela cirurgia, pode, se não houve um acompanhamento terapêutico satisfatório, começar a buscar novas vias de expressão. Observamos duas vias principais que podem se formar: a via depressiva e a via da compulsão.

A primeira começa com sintomas vagos: sensação de vazio, perda de interesse por coisas que anteriormente eram muito valorizadas, perda de eficiência no trabalho e angústia. Pode, nos casos mais graves, encaminhar para transtornos depressivos manifestos, como descritos na literatura ${ }^{22,23}$.

A segunda via que pode se abrir é a da compulsão. Vemos com muita frequência que passado o primeiro momento de triunfo sobre o sintoma, o traço impulsivo, existente já na base da personalidade do obeso começa, pouco a pouco, a se fazer presente e pressionar o indivíduo em direção ao alimento. Instala-se o terror e o fascínio pelas bolachas que dissolvem na boca, pelo biscoito de polvilho, pelo leite condensado, pelo sorvete e o chocolate. Uma verdadeira batalha interna se impõe. Um descuido e o peso começa a subir de novo. Disse uma das pacientes, no corredor do ambulatório, após deixar o grupo: "doutor, eu não sei mais o que faço, não tenho fome, mas fico o dia inteiro beliscando bolachas, e estou voltando a ganhar peso, mas não consigo parar". Essa fala vem fortemente marcada por uma angústia que transborda, em meio ao ar cansado de uma senhora visivelmente aprisionada numa batalha interna, sem descanso, ou seja, o comportamento compulsivo continua presente, e é a via do corpo, não do pensamento, que está disponível para essa paciente. Como proporcionar ou favorecer a atividade de pensamento para sujeitos que, durante quase toda sua existência, tiveram como via preferencial para lidar com conflitos e tensões internas o corpo? Está aí um grande desafio.

A característica peculiar da população atendida coloca a equipe de saúde frente a questões bastante particulares e 
que demandam atitudes terapêticas diferenciadas. Como a essência da estrutura psicológica do obeso mórbido é constituída por elementos primitivos, ligados a problemas em fases precoces do desenvolvimento emocional, com intensos sentimentos de ódio frente à frustração e à falta, vemos esse universo emocional interno transbordar para o ambiente do ambulatório e por vezes contaminar o próprio funcionamento da equipe e dos profissionais individualmente. Tal fenômeno ocorre a partir de identificações projetivas maciças ${ }^{24}$ de elementos psíquicos para dentro da equipe. Quantas vezes podemos facilmente identificar atitudes de rejeição a certos pacientes que voltam a engordar, ou que não conseguem cumprir corretamente a dieta, ou, então, em relação àqueles que passam a ter queixas em relação à cirurgia (dores, intolerância alimentar, etc.)? Acreditamos que esses sentimentos são a consequência da projeção maciça do mundo interno dos pacientes na equipe. Tal fato tem uma importância prática relevante, pois aponta para a necessidade de os membros da equipe de saúde serem orientados e, mais do que isso, de receberem suporte psicoterapêutico que os capacite a identificar e elaborar internamente essa forte carga de afetos que o paciente impõe. Caso esse cuidado não seja tomado, podemos incorrer em erros muito facilmente, pois o tipo de sentimentos e reações que o paciente obeso desperta são, muito frequentemente, de rejeição e discriminação ${ }^{25,26}$.

Desse modo, as reações emocionais que aparecem no campo de trabalho devem estar no foco da atenção psicológica da equipe multidisciplinar. As condutas de orientação, de esclarecimento e de apoio psicológicos são absolutamente necessárias dentro de um grupo de pacientes que funciona a partir de padrões psíquicos primitivos. Nesses, os sentimentos de desamparo e o vazio são muito frequentes no pós-operatório, principalmente no pós-operatório tardio, quando se acham já distantes do período inicial de lua-de-mel com a cirurgia e com a equipe. É fundamental que a equipe esteja instrumentalizada para lidar com aqueles aspectos da dinâmica emocional dos pacientes que irão contaminar o campo de trabalho da equipe com sentimentos de rejeição (muitas vezes encoberto por formações reativas do tipo "apego excessivo" ou "idealização" da equipe ou de membros dela), dependência e adesão. Essas reações, se não são percebidas, tendem a provocar reações na equipe, correndo o risco de levar o paciente-que é a parte mais fragilizada desse cenário - a reações de rejeição inconsciente à equipe e ao tratamento proposto e abandono do seguimento pós-operatório.

Grande parte da dificuldade em lidar com pacientes que funcionam no nível do corpo - e o obeso mórbido é um exemplo disto - é que sua comunicação se dá, prioritariamente, através de elementos não-verbais, que são mais sentidos do que compreendidos racionalmente ${ }^{11,24}$. Desse modo, qualquer tentativa de compreender e responder a essa comunicação a partir da razão tende a gerar uma repetição estéril e que deixa incompreendida a comunicação pré-verbal, o que leva a reações negativas, tanto dos pacientes como da equipe de saúde.

\section{Uma proposta de classificação das estruturas psico- lógicas dos obesos e seu manejo pós-operatório}

Essas experiências nos permitem propor algumas orientações para o funcionamento da equipe multidisciplinar. Em primeiro lugar, vemos como necessária a compreensão da estrutura mental daqueles sujeitos que procuram o serviço.

A partir da observação clínica dos pacientes e de referenciais psicodinâmicos, distinguimos três grupos de pacientes: os de estrutura melancólica, os de estrutura desmentalizada e os de estrutura perversa. Cada uma dessas estruturas apresenta distintos modos de enfrentar os desafios da cirurgia, com prognósticos e complicações pós-operatórias diferentes.

A partir da descrição da melancolia feita por Freud ${ }^{27}$, que seria uma condição na qual o sujeito passa a viver com a "sombra do objeto" perdido incrustada na própria constituição do Eu e da qual o sujeito não consegue se libertar, postulamos uma estrutura melancólica. Esses pacientes, após a cirurgia, terão grandes dificuldades em suportar a falta representada pela restrição alimentar com maior probabilidade de desenvolver quadros depressivos e ansiosos. São os mais propensos a desenvolver táticas de alimentação que compensem a restrição da cirurgia, ou seja, são os que "beliscam" o dia todo, compulsivamente, angustiam-se, mas não conseguem se controlar. A sensação de falta torna-se intolerável ao reativar a vivência de perda do objeto de amor. São os pacientes que respondem, ainda que pobremente, aos medicamentos antidepressivos.

A estrutura desmentalizada é, a nosso ver, um grande desafio no pós-operatório, pois são sujeitos que funcionam com muito pouco elemento mental para elaboração, e que, portanto, apresentam um contato muito pobre consigo mesmos e com os outros. Atualmente, muitos autores psicanalistas têm procurado fundamentar metapsicologicamente as patologias do vazio ou de déficit, entre elas a obesidade mórbida $^{28-31}$, nas quais existe uma pobreza de representações mentais, com grande prejuízo para a construção de símbolos e, consequentemente, para o pensamento elaborativo. Esses pacientes, por falta de elementos para a elaboração, têm como via preferencial de descarga pulsional o corpo. Para eles, as orientações técnicas e os esclarecimentos representam muito pouco ou não são aproveitados, por falta de elementos de escuta e de compreensão. Alguns continuam comendo quase como faziam antes da cirurgia, já alguns meses após o procedimento, num ato automático e pouco elaborado. O desafio que a cirurgia representa é muito pouco percebido. A participação desses sujeitos no grupo terapêutico é muito discreta, quase não falam, não emitem opiniões, permanecem atentos à conversa dos outros. $\mathrm{O}$ risco de não conseguirem se manter fiéis à proposta inicial, e mesmo de não conseguirem compreender a complexidade do processo de emagrecimento proposto, leva-os muito frequentemente a ganharem o peso perdido em poucos anos, representando o procedimento cirúrgico um risco desnecessário. 
Tal postura denuncia aquilo que chamamos de esvaziamento psíquico, e que é o que caracteriza as patologias de déficit. Devemos, pois, questionar a indicação cirúrgica para estes sujeitos, ou melhor dizendo, rever os critério para indicação cirúrgica, que talvez deveriam ser mais rígidos, limitando-se àqueles nos quais a obesidade causa grandes complicações para a saúde, com iminente risco de vida.

Por fim, baseados nas ideias de Freud sobre o fetichis$\mathrm{mo}^{32}$, propusemos uma estrutura perversa. Para esse autor existem alguns indivíduos que criam manobras para driblar a percepção dos limites que são impostos pela realidade. Ao se depararem com as inevitáveis faltas que a realidade continuamente impõe, agem como se elas não existissem e, por fim, os outros devem funcionar simplesmente como coadjuvantes dessa farsa da completude. São sujeitos que criam impasses, pois necessitam de que a realidade da falta seja o tempo todo burlada e, para isso, precisam da complacência dos outros que os rodeiam. Serão, portanto, aqueles pacientes que criarão problemas pós-cirúrgicos em função das exigências que farão à equipe multidisciplinar, sobretudo aos médicos. Como a via do corpo é a mais acessível, serão as queixas corporais as mais frequentes, tais como dores abdominais indefinidas, episódios de vômitos inexplicáveis clinicamente, intolerância alimentar, episódios bulímicos. São pacientes que causam as mais intensas reações emocionais negativas na equipe e mesmo nos outros pacientes. Exigem atenção contínua da equipe, estão constantemente se queixando, colocando a equipe como responsável pelo seu sofrimento, e passando sempre a mensagem que "agora que vocês me operaram, são responsáveis por minha insatisfação". A projeção de seu inconformismo com a realidade que os frustra na equipe é maciça, e geralmente a reação da equipe é de rejeição, quando não de uma agressão velada ao paciente. No grupo terapêutico são disruptivos, jogam o grupo contra o terapeuta e a equipe, "denunciam" as falhas do sistema de atendimento e de membros da equipe de trabalho. São aqueles que mais buscam a equipe de um modo invasivo e adesivo, o que desperta as mais intensas reações contratransferenciais negativas.

A partir dessas especulações teórico-clínicas, fica evidente que, idealmente, qualquer candidato a submeterse à cirurgia bariátrica deveria passar por um processo investigativo de sua estrutura mental e por uma abordagem psicoterapêutica profunda antes do procedimento cirúrgico. Mas isso tornaria o procedimento quase inviável pela sua complexidade, além da recusa da maioria dos pacientes, que querem uma solução rápida para seu problema. Essa realidade pode ser contornada pela presença, na equipe, de profissionais especialistas em saúde mental e com boa experiência em práticas psicodinâmicas, bem como em aplicação de técnicas psicoterapêuticas. Tais profissionais teriam como papel identificar previamente a estrutura mental de candidatos à cirurgia, propondo estratégias de abordagem psicológica pré e pós-operatórias individualizadas, visando evitar que complicações psicológicas viessem a tornar desnecessário todo o risco assumido pelos pacientes e pela equipe.

\section{Referências}

1. World Health Organization (WHO). Obesity and overweight. Disponível em: http://www.who.int/dietphysicalactivity/publications/facts/obesity/en/. Acessado ago 2006.

2. Buchwald H. Consensus conference statement bariatric surgery for morbid obesity: health implications for patients, health professionals, and third-party payers. J Am Coll Surg. 2005;200:593-604.

3. Hubbard VS, Hall WH. Gastrointestinal surgery for severe obesity. Obes Surg. 1991;1(3):257-65.

4. Segal A, Fandiño J. Indicações e contra-indicações para realização das operações bariátricas. Rev Bras Psiquiatr. 2002;24(Supl 3):S68-72.

5. Appolinário JC. Transtornos alimentares. In: Botega NJ, ed. Prática psiquiátrica no hospital geral: interconsulta e emergência. $2^{\mathrm{a}}$ ed. Porto Alegre: Artmed; 2006. p. 325-40.

6. Omalu BI, Cho P, Shakir AM, Agumadu UH, Rozin L, Kuller LH, et al. Suicides following bariatric surgery for the treatment of obesity. Surg Obes Relat Dis. 2005;1(4):447-9.

7. Leal CW, Baldin N. O impacto emocional da cirurgia bariátrica em pacientes com obesidade mórbida. Rev Psiquiatr RS. 2007;29(3):324-7.

8. Kalarchian MA, Marcus MD, Levine MD, Courcoulas AP, Pilkonis PA, Ringham RM, et al. Psychiatric Disorders Among Bariatric Surgery Candidates: Relationship to Obesity and Functional Health Status. Am J Psychiatry. 2007;164(2):328-34.

9. American Psychiatry Association. Diagnostic and statistical manual of mental disorders, DSM-IV-TR. 4th ed. Washington, DC: American Psychiatry Association. 2000.

10. Magdaleno R Jr., Chaim EA, Turato ER. Understanding life experiences of Brazilian women after bariatric surgery: a qualitative study. Obes Surg. 2008. [Epub ahead of print].

11. Mahler M. O processo de individuação e separação. Porto Alegre: Artmed; 1982.

12. Tustin F. Barreiras autistas em pacientes neuróticos. Porto Alegre: Artmed; 1990.

13. Freud S. Três ensaios sobre a teoria da sexualidade. In: Freud S. Obras Completas, vol. VII. $2^{a}$ ed. Rio de Janeiro: Imago; 1987. p. 129-237.

14. Freud S. O Instinto e suas vicissitudes. In: Freud S. Obras completas. vol. 9. Rio de Janeiro: Imago; 1974. p. 129-62.

15. Winnicott DW. O papel de espelho da mãe e da família no desenvolvimento infantil. In: Winnicott DW. O brincar e a realidade. Rio de Janeiro: Imago;1975. p. 153-62.

16. Winnicott DW. A mente e sua relação com o psicossoma. In: Winnicott DW. Da pediatria à psicanálise: obras escolhidas. Rio de Janeiro: Imago; 2000. p. 332-46.

17. Turato ER. Tratado da metodologia da pesquisa clínico-qualitativa: construção teórico-epistemológica, discussão comparada e aplicação às áreas da saúde e humanas. Petrópolis: Vozes; 2003.

18. Arruda SLS. Vivências clínicas de um grupo de mães cujos filhos estão em ludoterapia [tese]. Campinas: Universidade Estadual de Campinas; 1991.

19. Fontanella BJB. Procura de tratamento por dependentes de substâncias psicoativas: um estudo clínico - qualitativo [tese]. Campinas: Universidade Estadual de Campinas; 2000.

20. Glinski J, Wetzler S, Goodman E. The psychology of gastric bypass surgery. Obes Surg. 2001;11(5):581-8.

21. DeMaria EJ. Bariatric surgery for morbid obesity. N Engl J Med. 2007;356(21):2176-83.

22. Dixon JB, Dixon ME, O'Brien PE. Depression in association with severe obesity: changes with weight loss. Arch Intern Med. 2003;163(17):2058-65.

23. Roberts RE, Kaplan GA, Shema SJ, Strawbridge WJ. Are the obese at greater risk for depression? Am J Epidemiol. 2000;152(2):163-70.

24. Klein M. Notas sobre alguns mecanismos esquizóides. In: Klein M. Inveja e gratidão e outros trabalhos. Rio de Janeiro: Imago; 1991. p. 17-43.

25. Bocchieri LE, Meana M, Fischer BL. A review of psychosocial outcomes of surgery for morbid obesity. J Psychosom Res. 2002;52(3):155-65.

26. Kaminsky J, Gadaleta D. A study of discrimination within the medical community as viewed by obese patients. Obes Surg. 2002;12(1):14-8.

27. Freud S. Luto e melancolia. In: Freud S. Obras completas. vol. 9. Rio de Janeiro: Imago; 1974. p. 271-291.

28. Levy R. A visão topográfica no processo psicanalítico: o irrepresentável. Rev Bras Psicanal. 2003;37(4):1067-77.

29. Botella C, Botella S. Irrepresentável: mais além da representação. Porto Alegre: Criação Humana; 2002.

30. Magdaleno Jr R. A dialética de Eros e o mal-estar na cultura hoje. Ide. 2008;31(46):46-51.

31. Lisondo ABD. Na cultura do vazio, patologias do vazio. Rev Bras Psicanal. 2004;38(2):335-58.

32. Freud S. Fetichismo. In: Freud S. Obras completas. vol. 9. Rio de Janeiro: Imago; 1974. p. 175-85. 